D.O.I.: $10.3895 / \mathrm{S} 1808-04482010000300004$

\title{
APLICACIÓN DE LA TEORÍA DE COLAS Y DE LA SIMULACIÓN AL EMBARQUE DE MINERAL DE HIERRO Y MANGANESO EN LA TERMINAL MARÍTIMA DE PONTA DA MADEIRA
}

\section{APPLICATION OF QUEUEING THEORY AND SIMULATION TO SHIPMENT OF IRON ORE AND MANGANESE IN THE PONTA DA MADEIRA MARITIME TERMINAL}

\author{
Gustavo Rossa Camelo ${ }^{1}$; Antônio Sérgio Coelho ${ }^{2}$; Renata Massoli Borges ${ }^{3}$ \\ ${ }^{1}$ Universidade Federal de Santa Catarina - UFSC - Florianópolis - Brasil \\ gustavorcamelo@hotmail.com \\ ${ }^{2}$ Universidade Federal de Santa Catarina - UFSC - Florianópolis - Brasil \\ coelho@deps.ufsc.br \\ ${ }^{3}$ Universidade do Sul de Santa Catarina - UNISUL - Florianópolis - Brasil \\ renata@fama.br
}

\begin{abstract}
Resumen
De entre las principales técnicas disponibles utilizadas en procesos de análisis y resolución de problemas sobresalen la Teoría de Colas y la Teoría de la Simulación: aquella, un método analítico para abordaje del asunto; esta, una técnica de modelaje que busca mejor representar el sistema en estudio. El uso de estas técnicas de modelaje, para resoluciones de problemas complejos $y$ dinámicos, tiene crecido mucho en los últimos años, aunque, hay una carencia perceptible en la literatura de aplicaciones en situaciones reales. En contribución, este trabajo investiga el uso de la Teoría de Colas y de la Simulación aplicadas al embarque de mineral de hierro y manganeso en la Terminal Marítima de Ponta da Madeira - TMPM, perteneciente a Vale. Con la Teoría de Colas se pretende analizar características de atendimiento a los buques que atracan en el Pier I y Pier III para cargamento de mineral de hierro y manganeso, tales como: número medio de buques en la cola y en el sistema, tiempo medio durante el cual el buque permanece en la cola y en el sistema etc. $Y$, con la técnica de la Simulación se pretende simular la operación del Pier IV, que consumirá $R \$ 2$ mil millones en inversión, aumentará la capacidad de la terminal en 100 millones de toneladas al año y que tendrá capacidad de cargar 53 buques por mes.
\end{abstract}

Palabras-clave: Teoría de Colas. Simulación. TMPM.

\section{Introducción}

En la actual coyuntura económica el Brasil es una de las economías que más crecen en el mundo. Esta ascensión brasileña en los últimos años coloca el país como uno de los grandes destaque del actual escenario económico mundial. De acuerdo con analistas, la ascensión de Brasil, Rusia, India y China - el llamado BRIC, grupo de países que forman el pelotón de elite de las economías emergentes - cambiará el rumbo de la economía mundial. 
En el Brasil, empresas y sectores enteros tiene se beneficiado con la onda de prosperidad que el país tiene pasado en los últimos años. La Vale, empresa de minería privatizada en 6 de mayo de 1997, fue una de las inúmeras empresas brasileñas que supieron tirar provecto de este actual momento, transformándose en el mayor fenómeno del capitalismo brasileño, presentando un ritmo avasallador de crecimiento.

La Vale es la mayor empresa privada de la América Latina y mayor productora de mineral de hierro del mundo. Su estructura integrada de logística es formada por cerca de 10 mil quilómetros de malla ferroviaria, cinco terminales portuarios - localizados en Espírito Santos, Sergipe y Maranhão - y una terminal carretera-ferrocarril.

En números, la Vale es responsable por $16 \%$ del movimiento de cargas en el Brasil y $30 \%$ del movimiento portuario brasileño. El sistema logístico de la Vale se divide en: Sistema Sur, compuesto por la Ferrovía Vitória a Minas (EFVM), Ferrovía Centro Atlántica (FCA), Puertos Sur - en Vitória (ES), la Terminal de Productos Diversos (TPD), la Terminal de Graneles Líquidos (TGL) y la Terminal de Praia Mole (TPM) y en Barra dos Coqueiros (SE), la Terminal Marítima Inácio Barbosa (TMIB); y, el Sistema Norte, compuesto de la Ferrovía Carajás (EFC), Ferrovía Norte Sul (FNS) y Terminal Marítima de Ponta da Madeira (TMPM).

En el Sistema Sur el mineral de hierro producido en el Cuadrilátero Ferrífero, en Minas Gerais es transportado para el Complejo Portuario de Tubarão, en Vitória (ES), y para el Puerto de Itaguaí, en el Rio de Janeiro (RJ); mientras, en el Sistema Norte el mineral producido en Carajás, en el Pará, es transportado para la Terminal Marítima de Ponta da Madeira, en São Luís (MA).

La TMPM, objeto de estudio de este trabajo, es el segundo en movimiento de cargas en el Brasil, con un flujo anual de 72.941.142 toneladas, atrás apenas del puerto de Tubarão también administrada por la Vale, de acuerdo con el Anuario EXAME de Infra-Estructura 2008-2009.

Este artículo pretende a través de la Teoría de Colas analizar características de atendimiento a los buques que atracan para cargamento de mineral de hierro y manganeso. Para tal operación tiene disponible tres muelles, uno en el Pier I con capacidad de cargamento de 16.000 ton/h y dos en el Pier III con capacidad de 8.000 ton/h cada.

Conjuntamente, a través de la teoría de la Simulación, este artículo pretende simular la operación del Pier 4, que está en fase de implantación y que consumirá R \$ 2 mil millones en inversión, aumentará la capacidad de la terminal en 100 millones de toneladas por año y que tendrá capacidad de cargar 53 buques por mes.

\section{Terminal marítima de ponta da madeira}

Inaugurado en 1986 fue inicialmente proyectado para operar con mineral de hierro y manganeso principalmente destinado a la exportación, mas desde temprano demostró su inclinación 
para ser grande (VALE, 2005).

Escogido como término del Ferrocarril Carajás, es responsable por el transporte de mineral de hierro, pelotas, cobre, manganeso, hierro gusa y soya para el exterior, principalmente para la Europa y el Asia Oriental.

La TMPM es responsable por el transporte de $86 \%$ de toda la carga movida en el Maranhão. Son tres pieres, con profundidades de 23m (Pier I - uno de los más profundos del mundo), 18m (Pier II) y $21 \mathrm{~m}$ (Pier III). Es el único del País capaz de cargar totalmente el mayor granelero del mundo, el Berge Stahl, con capacidad para transportar 364.767 toneladas (ABRETI, 2007).

Es considerado el mejor puerto del país de acuerdo con investigación realizada por COPPEAD/UFRJ junto a las empresas industriales exportadoras, agentes y armadores, terminales y administraciones portuarias. Según usuarios oídos por la investigación, su profundidad y la agilidad de las operaciones están entre los motivos que colocaron la Ponta da Madeira en lugar de destaque. Es una isla de excelencia en medio al caos que puede provocar el naufragio del sistema portuario brasileño (EXAME, 2007).

Para hacer justicia al título de mejor del País, el puerto recibirá inversiones de peso en la expansión de la capacidad que deberá en 2011 alcanzar 200 millones de toneladas.

\section{Teoría de colas}

Todas las personas ya pasaran por el aborrecimiento de tener que esperar en colas para un atendimiento. Las colas pueden ocurrir en el desenvolvimiento de cualquier actividad humana y todos nosotros, por experiencias cotidianas, las conocemos. Inclusive, las colas representan un de los síntomas más visibles de funcionamiento deficiente de un sistema. A pesar de causar enfado y prejuicios, tenemos que convivir con colas en la vida real, visto que es económicamente inviable súper dimensionar un sistema para que nunca existan colas. Lo que se pretende es obtener un balanceo adecuado que permita un atendimiento aceptable que satisfaga la relación costo-beneficio (ANDRADE, 2004; COSTA, 2004; PORTUGAL, 2005; PRADO, 2006).

Ejemplos reales de aplicación de esa técnica pueden ser encontrados en las más diversas áreas, tales como telecomunicaciones; ingeniería de tráfico; bancos; call center; hospitales; aeropuertos; restaurantes; supermercados; programación de ómnibus (por ejemplo, BROWN et al., 2002; BRUIN et al., 2005; GREEN, 2006; JOSHI et al., 1992; MORE, 1977; XIAO e ZHANG, 2010).

Las formaciones de colas ocurren cuando la procura por determinado servicio es superior a la capacidad de atendimiento del sistema. De esta forma, la Teoría de Colas por medio de fórmulas matemáticas tienta encontrar un punto de equilibrio que satisfaga el cliente y que sea económicamente viable para el prestador del servicio. 
La Teoría de Colas es un método analítico, desarrollado por A. K. Erlang (1909), que estudia la formación de colas y sus características por medio de fórmulas matemáticas.

Un sistema de colas puede ser descripto como clientes llegando a la procura de un servicio, esperando en cola, si no fueren atendidos inmediatamente, y saliendo del sistema después de atendidos.

Figura 1 - Representación de un sistema de colas

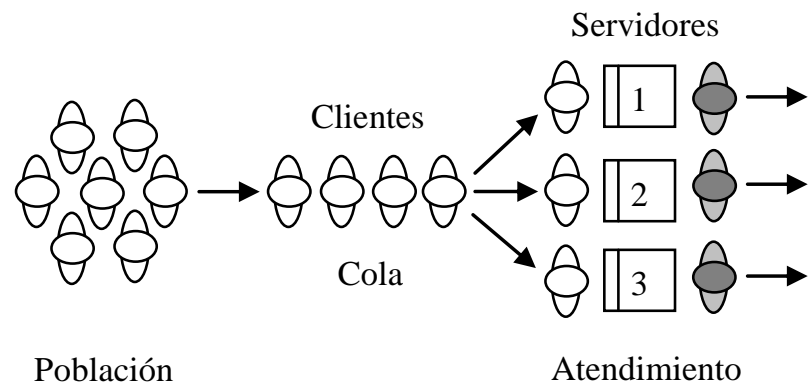

La Tabla 1 presenta y evalúa los principales elementos de un sistema de colas en observación:

Tabla 1 - Elementos de un sistema de colas

\begin{tabular}{|c|l|}
\hline Cliente & $\begin{array}{l}\text { Es la unidad que requiere atendimiento, pudendo ser máquina, personas, y } \\
\text { en este trabajo específico, buques. }\end{array}$ \\
\hline Cola & $\begin{array}{l}\text { Representa los clientes que esperan para ser atendidos. Normalmente no } \\
\text { incluyen los clientes siendo atendidos. }\end{array}$ \\
\hline $\begin{array}{c}\text { Canal de } \\
\text { atendimiento }\end{array}$ & $\begin{array}{l}\text { Proceso o sistema que realiza el atendimiento al cliente. Puede ser un } \\
\text { canal múltiplo o único. }\end{array}$ \\
\hline
\end{tabular}

\subsection{Características básicas}

En general, son seis características básicas del proceso de colas (ABAD, 2002; ANDRADE, 1994; BANKS et al, 1999; FARRERO et al, 2009; GROSS; HARRIS, 1974; MIRANDA, 2005; MIRSHAWKA, 1979; PRADO, 1999): proceso de llegada de los clientes; patrones de servicios de los asistentes; disciplina de cola; número de canales de servicios; capacidad de almacenamiento del sistema; número de etapas del servicio.

\subsubsection{Proceso de llegada de los clientes}

El proceso de llegada del cliente o entrada en el sistema de colas es medido en razón del número medio de llegadas por una dada unidad de tiempo ( $\lambda$ - tasa media de llegada) o por el tiempo medio entre llegadas sucesivas (IC - intervalo medio de tiempo entre llegadas).

\subsubsection{Patrones de servicio de los asistentes}


El patrón de servicio es descrito por la tasa de servicio $(\mu-$ número de clientes en atendimiento en un determinado intervalo de tiempo) o por el tiempo de servicio (TA - tiempo necesario para atender el cliente).

\subsubsection{Disciplina de Cola}

Se refiere a la manera como los clientes son escogidos para entrar en servicio después de una cola ser formada. La disciplina más común se da por la orden de llegada, en la cual el primero que llega es el primero a ser atendido (First in, First out - FIFO o PLPS). Otras alternativas son: el último a llegar es el primero a ser atendido (Last in, First out - LIFO o ULPS); por el atendimiento con prioridad para ciertas clases de clientes, independiente de la hora de llegada en el sistema, muy común para viejos y grávidas (Priority service - PRI); y por la selección de atendimiento de forma aleatoria, independiente de la orden de llegada en la cola (Service in randon order - SIRO).

\subsubsection{Número de canales de servicios}

Se refiere al número de servidores en paralelo que prestan servicios simultáneos a los clientes. Un sistema de colas pode presentar un o múltiplos canales de atendimiento, operando independientemente uno del otro. En ese último caso, puede se tener una cola única o una cola para cada canal.

La Figura 2 y Figura 3 presentan las estructuras típicas de sistemas de colas:

Figura 2 - Estructura de los sistemas de colas

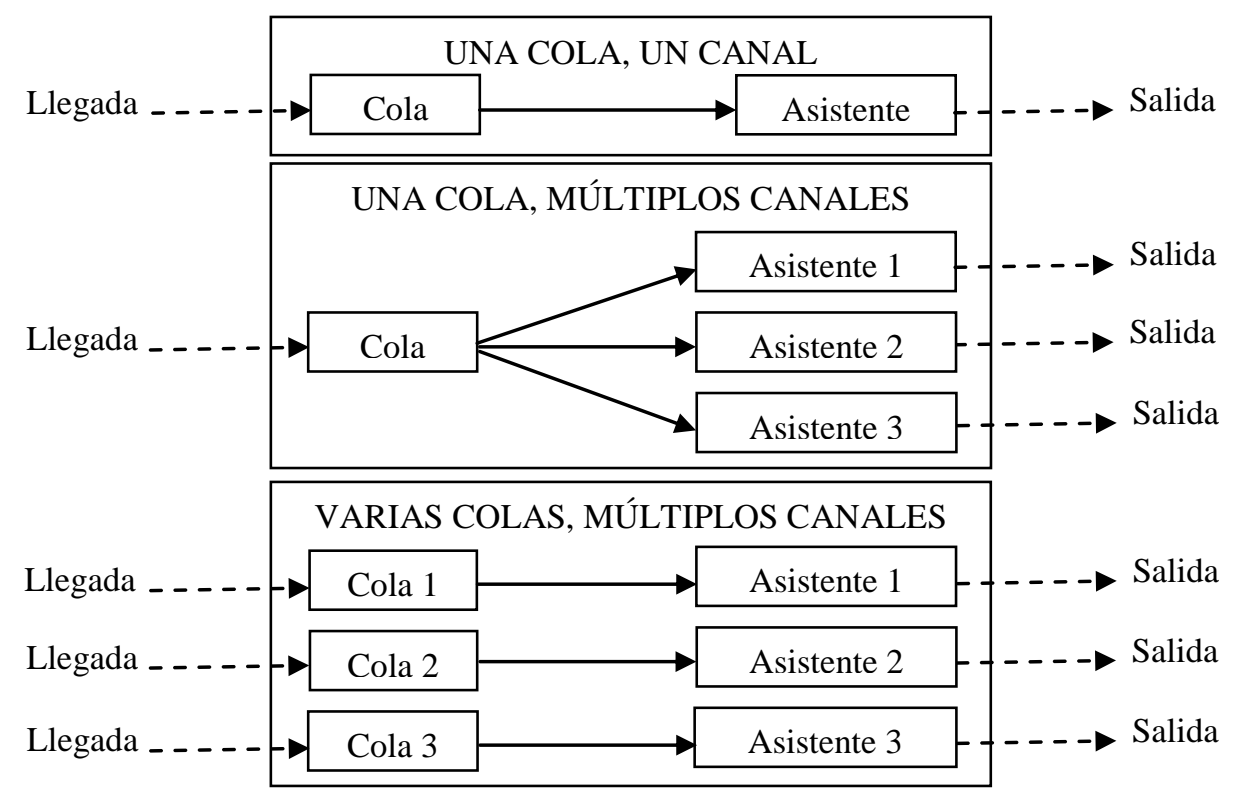

\subsubsection{Capacidad de Almacenamiento del Sistema}

Es el número máximo de usuarios, tanto aquellos siendo atendidos cuanto aquellos en las 
colas, permitidos en el estabelecimiento de prestación de servicios al mismo tiempo, pudendo ser finito o infinito.

\subsubsection{Etapas del Servicio}

Un sistema de colas puede tener apenas una única etapa de atendimiento, como en el caso de supermercados y peluquerías, o puede tener varias etapas. En ese caso, denominado de múltiples etapas, el cliente precisa pasar por varias etapas hasta dejar el sistema.

Figura 3 - Sistema múltiples etapas de colas

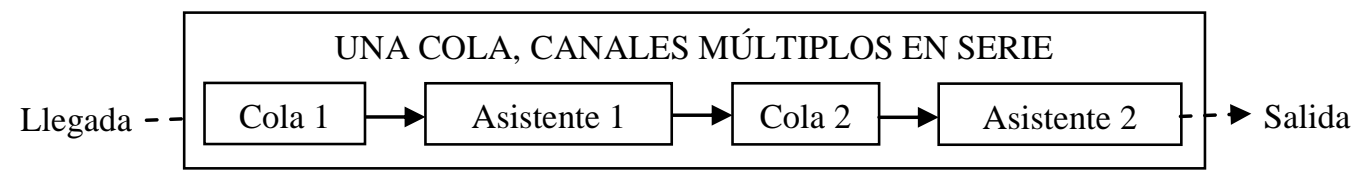

\subsection{Variables aleatorias fundamentales}

Un sistema de colas, en situación estable, en la cual clientes llegan y esperan para ser atendidos por "c" servidores, conforme representado por la Figura 4, presenta las siguientes variables aleatorias:

Figura 4 - Variables en un sistema de colas

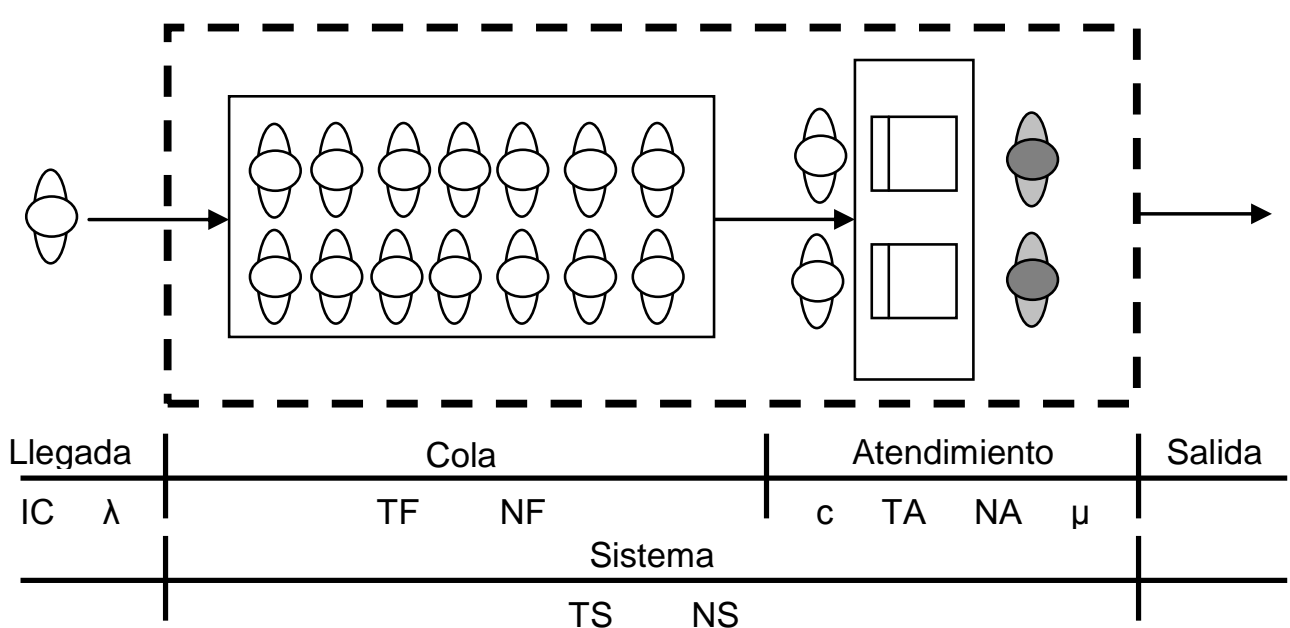

Fuente: Adaptado de Prado (2006, p. 39).

- Variables referentes al proceso de llegada

$\lambda=$ tasa media de llegada o ritmo medio de llegada;

$\mathrm{IC}=$ intervalo medio entre llegadas.

- Variables referentes con la cola

$\mathrm{TF}=$ tiempo medio de permanencia en la cola;

$\mathrm{NF}=$ número medio de clientes en la cola. 
- Variables referentes al proceso de atendimiento o de servicio

$\mathrm{TA}=$ tiempo medio de atendimiento o de servicio;

$\mathrm{c}=$ capacidad de atendimiento o cuantidad de servidores (asistentes);

$\mathrm{NA}=$ número medio de transacciones o clientes que están siendo atendidos;

$\mu=$ tasa media de atendimiento o ritmo medio de atendimiento de cada servidor.

- Variables referentes al sistema

$\mathrm{TS}=$ tiempo medio de permanencia en el sistema;

$\mathrm{NS}=$ número medio de transacciones o clientes en el sistema.

La Tabla 2 muestra las relaciones básicas entre las variables de un sistema de colas:

Tabla 2 - Relaciones básicas entre variables

\begin{tabular}{|l|l|}
\hline \multicolumn{1}{|c|}{ VARIABLES } & \multicolumn{1}{c|}{ FÓRMULA } \\
\hline Intervalo entre Llegadas & $\mathrm{IC}=1 / \lambda$ \\
\hline Tiempo del Atendimiento & $\mathrm{TA}=1 / \mu$ \\
\hline Tasa de Utilización de los Asistentes & $\rho=\lambda / \mathrm{c} \mu$ \\
\hline Intensidad del Tráfico & $\mathrm{i}=|\lambda / \mu|=|\mathrm{TA} / \mathrm{IC}|$ \\
\hline & $\mathrm{NS}=\mathrm{NF}+\mathrm{NA}$ \\
Relaciones entre Cola, Sistema y & $\mathrm{NA}=\lambda / \mu$ \\
Atendimiento & $\mathrm{NS}=\mathrm{NF}+\lambda / \mu=\mathrm{NF}+\mathrm{TA} / \mathrm{IC}$ \\
& $\mathrm{TS}=\mathrm{TF}+\mathrm{TA}$ \\
& $\mathrm{NA}=\rho=\lambda / \mathrm{c} \mu$ \\
\hline \multirow{2}{*}{ Fórmulas de Little } & $\mathrm{NF}=\lambda . \mathrm{TF}$ \\
\hline \multirow{2}{*}{ Ciclo } & $\mathrm{NS}=\lambda . \mathrm{TS}$ \\
\hline
\end{tabular}

Fuente: Prado (2006, p. 43).

\subsection{Medidas de desempeño}

Son tres las medidas de desempeño que buscan reflejar la eficiencia de un sistema de colas en estudio: 1) las relacionadas al tiempo de espera del cliente en la cola y en el sistema; 2) las relacionadas al número de clientes en la cola y en el sistema; 3) las asociada con el uso y tiempo de inactividad de los servidores. Estas medidas son las siguientes:

Tabla 3 - Relaciones básicas entre variables

\begin{tabular}{|c|}
\hline \multicolumn{1}{c|}{ Referentes al sistema } \\
\hline $\mathrm{TS}=$ Tiempo medio que el cliente gasta en el sistema \\
$\mathrm{NS}=$ Número medio de clientes en el sistema \\
\hline \multicolumn{1}{c|}{ Referentes a la fila } \\
\hline $\mathrm{TF}=$ Tiempo medio que el cliente gasta en la cola de espera \\
$\mathrm{NF}=$ Número medio de clientes en la cola o tamaño medio de la cola \\
\hline Referente a utilización y al tiempo ocioso de los servidores \\
\hline $\mathrm{P}_{0}=$ Índice de ociosidad de las instalaciones \\
$\rho=$ Tasa de utilización de los asistentes \\
\hline
\end{tabular}

Fuente: Prado (2006, p. 43). 


\subsection{Modelos de colas}

En general, un modelo de colas puede ser descrito por la notación de Kendall: A/B/c/K/m/Z, conforme la tabla 4, en que: A describe la distribución de los intervalos entre llegadas; B describe la distribución del tiempo de servicio; c es el número de canales de servicios o capacidad de atendimiento; $\mathrm{K}$ es el número máximo de clientes permitidos en el sistema; $\mathrm{m}$ es el tamaño da población que proveer clientes y $\mathrm{Z}$ es la disciplina de la cola.

Tabla 4 - Procesos de colas

\begin{tabular}{|c|c|c|c|}
\hline \multicolumn{2}{|r|}{ Características } & Símbolos & Explicación \\
\hline A & $\begin{array}{c}\text { Distribución de tiempo } \\
\text { entre llegadas }\end{array}$ & $\begin{array}{l}\mathrm{M} \\
\mathrm{D} \\
\mathrm{E} \delta \\
\mathrm{GI}\end{array}$ & $\begin{array}{l}\text { Ley de Poisson (Markoviano) } \\
\text { Determinística } \\
\text { Erlang } \delta(\delta=1,2, \ldots) \\
\text { Independencia General }\end{array}$ \\
\hline B & $\begin{array}{c}\text { Distribución de tiempo } \\
\text { de servicio }\end{array}$ & $\begin{array}{l}\text { M } \\
\mathrm{D} \\
\mathrm{E} \delta \\
\mathrm{GI}\end{array}$ & $\begin{array}{l}\text { Ley de Poisson (Markoviano) } \\
\text { Determinística } \\
\text { Erlang } \delta(\delta=1,2, \ldots) \\
\text { Independencia General }\end{array}$ \\
\hline $\mathrm{c}$ & $\begin{array}{l}\text { Número de canales de } \\
\text { servicios }\end{array}$ & $1,2, \ldots$, infinito & (2) \\
\hline $\mathrm{K}$ & Capacidad del sistema & $1,2, \ldots$, infinito & - \\
\hline $\mathrm{m}$ & Tamaño da población & $1,2, \ldots$, infinito & - \\
\hline $\mathrm{Z}$ & Disciplina de cola & $\begin{array}{c}\text { FIFO } \\
\text { LIFO } \\
\text { SIRO } \\
\text { PRI }\end{array}$ & $\begin{array}{l}1^{\circ} \text { que llega es el } 1^{\circ} \text { en ser atendido } \\
\text { Ultimo que llega es el } 1^{\circ} \text { en ser atendido } \\
\text { Servicio con orden aleatoria } \\
\text { Prioridad }\end{array}$ \\
\hline
\end{tabular}

Fuente: Adaptado de Portugal (2005, p. 20).

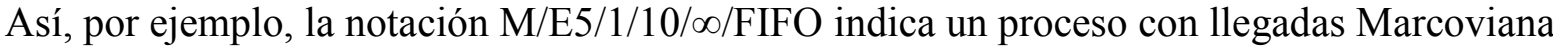
(Exponencial negativa o Poisson), atendimiento Erlang de quinto grado, 1 asistente, capacidad máxima del sistema igual a 10 clientes, población infinita y el primero que llega es el primero en salir del sistema.

\section{Simulación}

La simulación es una técnica de la Investigación Operativa que permite “imitar" el funcionamiento de un sistema real. Simular significa reproducir el funcionamiento de un sistema, con el auxilio de un modelo.

Para Prado (2006, p. 19): “[...] la modelaje de colas puede ser analizada por el ángulo de la simulación, en que no más se usan fórmulas matemáticas, pero apenas trata de imitar el funcionamiento de un sistema real a través de recursos computacionales".

La simulación es una herramienta poderosa de análisis que pretende determinar el mejor sistema para ser implementado o mejorado, permitiendo cuantificar los efectos de varias mudanzas en el sistema, siendo muy usado en situaciones en que es muy caro o difícil el experimento en la situación real. 
Para Andrade (2004, p. 113), se puede enumerar diversas razones para justificar el uso de la simulación en las más diversas áreas. Entre ellas se destacan:

1. Por medio de la simulación pueden ser estudiadas algunas variaciones en el medio ambiente y verificados sus efectos en todo el sistema;

2. La simulación posibilita el estudio y la experimentación de complejas interacciones internas de un determinado sistema;

3. Algunas técnicas analíticas exigen un avanzado conocimiento matemático, tanto para uso cuanto para comprensión. Una simulación de computador puede exigir poca o ninguna matemática compleja y, por lo tanto, puede ser intuitivamente fácil de comprender;

4. La simulación de sistemas complejos puede fornecer valiosa introvision en el sentido de descubrir las variables más importantes del sistema y la manera cómo interactúan;

5. La simulación permite la inclusión de complicaciones del mundo real;

6. La simulación no interfiere con los sistemas del mundo real;

7. Puede servir como un primero test para delinearen nuevas políticas y reglas de decisión para la operación de un sistema, antes de experimentar en el sistema real.

Cuanto a la forma de uso, la simulación normalmente está relacionada al empleo de ordenadores, aunque, también puede ser desarrollada manualmente y sin el uso de ese equipamiento.

Algunos lenguajes son mundialmente conocidas, como ARENA, AUTOMOD, CINEMA, GASP, GPSS, HOCUS, SIMAN, SIMSCRIPT, PROMODEL, TAYLOR, etc.

\section{Estudio de caso: embarque de mineral de hierro y manganeso en la terminal marítima de ponta da madeira}

El embarque de mineral de hierro (pellet feed, sinter feed, granulado y pelota) y manganeso por la Vale, en el corredor Norte (Carajás - São Luís) es realizado en tres muelle, un en el Pier I con capacidad de cargamento de 16.000 ton/h y dos en el Pier III (apodado de Norte y Sur) con capacidad de 8.000 ton/h cada. El Pier II, localizado dentro de la estructura del Puerto del Itaqui, es destinado para el embarque de soya y gusa.

La TMPM tiene tres canales de servicios con características de cola en paralelo, pues, cada muelle presenta características únicas de: Longitud, Calado, Air draft (distancia entre la línea de agua y la lanza del cargador de buques), TPB máximo, Capacidad de embarque etc. De esta forma, este trabajo irá analizar caso por caso (muelle por muelle) la capacidad de atendimiento a los buques. 
Tabla 5 - Características de la TMPM

\begin{tabular}{cccc}
\hline Características & Pier I & Pier II & Pier III \\
\hline Longitud: & $490 \mathrm{~m}$ & $280 \mathrm{~m}$ & $571 \mathrm{~m}$ \\
Calado: & $23 \mathrm{~m}$ & $18 \mathrm{~m}$ & $21 \mathrm{~m}$ \\
Air Draft: & $22,4 \mathrm{~m}$ & $18 \mathrm{~m}$ & $22.4 \mathrm{~m}$ \\
TPB Máximo: & 420.000 & 150.000 & 200.000 \\
Capacidad de & $16.000 \mathrm{t} / \mathrm{h}$ & $1.500 \mathrm{t} / \mathrm{h}$ (soya) & $8.000 \mathrm{t} / \mathrm{h}$ \\
Embarque: & & $2.00 \mathrm{t} / \mathrm{h}$ (gusa) & \\
\hline
\end{tabular}

Fuente: VALE (2009).

Con base en los datos de atraques y desatraques de la Terminal Marítima de Ponta da Madeira durante los meses de agosto, setiembre, octubre de 2009, se puede considerar que:

Tabla 6 - Datos de atraques y desatraques (en días)

\begin{tabular}{c|c|c|c}
\hline & Pier I & $\begin{array}{c}\text { Pier III - } \\
\text { Norte }\end{array}$ & $\begin{array}{c}\text { Pier III - } \\
\text { Sur }\end{array}$ \\
\hline Ritmo Medio de Llegada $(\lambda)$ & 0,63 & 0,59 & 0,58 \\
Intervalo Medio entre Llegadas (IC) & 1,59 & 1,69 & 1,73 \\
Ritmo Medio de Atendimiento $(\mu)$ & 0,74 & 0,73 & 0,69 \\
Tiempo Medio de Atendimiento (TA) & 1,36 & 1,37 & 1,44 \\
\hline
\end{tabular}

Fuente: VALE (2009)

Con base en los datos obtenidos se puede definir el problema de colas como siendo del tipo $\mathrm{M} / \mathrm{M} / 1 / \infty / \infty / \mathrm{FIFO}$, o sea, las llegadas y lo atendimiento son marcovianos (siguen la Distribución de Poisson o la Exponencial Negativa), hay apenas un único canal de atendimiento, no hay restricción de capacidad y de populación y la orden de atendimiento del tipo FIFO.

La Tabla 7 presenta las principales variables utilizadas como medidas de desempeño en un sistema de colas con un único asistente.

Tabla 7 - Fórmulas para un único asistente

\begin{tabular}{|c|c|c|}
\hline Nombre & Descripción & Fórmula \\
\hline$\rho$ & Tasa de Utilización & $\rho=\frac{\lambda}{\mu}$ \\
\hline NF & Número Medio de Clientes en la Cola & $N F=\frac{\lambda^{2}}{\mu(\mu-\lambda)}$ \\
\hline NS & Número Medio de Clientes en el Sistema & NS $=\frac{\lambda}{\mu-\lambda}$ \\
\hline TF & $\begin{array}{l}\text { Tempo Medio durante o cual o Cliente permanece en la } \\
\text { Cola }\end{array}$ & $T F=\frac{\lambda}{\mu(\mu-\lambda)}$ \\
\hline $\mathrm{TS}$ & $\begin{array}{l}\text { Tempo Medio durante el cual el Cliente permanece en el } \\
\text { Sistema }\end{array}$ & $T S=\frac{1}{\mu-\lambda}$ \\
\hline $\mathrm{P}_{0}$ & Probabilidad de existieren n Clientes en el Sistema & $n=\left(1-\frac{\lambda}{u}\right)\left(\frac{\lambda}{u}\right.$ \\
\hline
\end{tabular}

Con la posesión de los datos de atraques y desatraques se aplicó estos a las fórmulas presentadas y los resultados obtenidos son presentados en la Tabla 8. 
Tabla 8 - Resultado Obtenido

\begin{tabular}{|c|c|c|c|}
\hline \multicolumn{4}{|c|}{ MEDIDAS DE DESEMPEÑ - TERMINAL MARÍTIMA DE PONTA DA MADEIRA } \\
\hline Medida & Pier I & Pier III - Norte & Pier III - Sur \\
\hline$\rho$ & $0,854 \cong 85 \%$ & $0,813 \cong 81 \%$ & $0,836 \cong 84 \%$ \\
\hline NF & 5,0 & 3,5 & 4,3 \\
\hline NS & 5,9 & 4,3 & 5,1 \\
\hline TF & 8,0 & 5,9 & 7,4 \\
\hline TS & 9,3 & 7,3 & 8,8 \\
\hline $\mathrm{P}_{0}$ & $15 \%$ & $19 \%$ & $16 \%$ \\
\hline
\end{tabular}

$\mathrm{Al}$ analizar los resultados obtenidos es evidente la eficiencia del terminal, que presenta una tasa media de ocupación $(\rho)$ de los muelles superior al 80\%. La mayor tasa de ocupación es observada en lo Pier I. Este, debido a sus características, es el único con capacidad de recibir los grandes graneleros (de más de 200.000 TPB). Al concentrar la demanda de los grandes buques que cargan en el terminal, el Pier I presenta las siguientes medidas de desempeño: mayor tasa de ocupación (85\%), mayor tiempo medio en el cual el cliente permanece en la cola (8 días) y en el sistema (9,3 días), y la menor probabilidad de no existir ningún cliente en el sistema (15\%).

El Pier III - Norte tiene una tasa de ocupación de $81 \%$. Esto presenta un número medio de 3,5 buques en la cola y 4,3 buques en el sistema aguardando por atendimiento y el tiempo medio gasto por un buque en la cola y en el sistema es respectivamente, 5,9 e 7,3 días.

El Pier III - Sur tiene una tasa de ocupación de 84\%. Esto presenta un número medio de 4,3 buques en la cola y 5,1 en el sistema. Un buque programado para atracar en el Pier III - Sur gasta en media 7,4 días en cola y 8,8 días en el sistema.

A través del uso de la simulación computacional tiene que para el período de un año 661 buques pasaran por el sistema, resultando una media de 55 buques por mes. El Pier I fue responsable por el cargamento de 218 buques aunque el Pier III - Norte cargó 235 y el Pier III - Sur cargó 219.

Con una tasa de ocupación muy alta, la terminal está operando en el límite de la su capacidad operacional. De esta forma para garantir la producción y transporte de los minerales en el medio y largo plazo la Vale irá ampliar la capacidad actual de la Terminal Marítima de Ponta da Madeira. Para tal, irá construir un nuevo Pier para cargamento de mineral de hierro y manganeso, el Pier IV.

El objetivo de la mineradora es ampliar en 100 millones de toneladas al año la capacidad de la terminal. Con profundidad mínima de 25 metros, el Pier IV tendrá dos muelles para atraque y capacidad para recibir buques hasta 400 mil toneladas de porte bruto (TPB). Con una puente de acceso de 1.620 metros, tendrá capacidad de cargamento de dos buques simultáneamente en un total de 53 buques por mes (EXAME, 2009).

El Pier IV tendrá estructura similar al Pier III, poseyendo dos muelles para atraques y 
desatraques, pero, con características del Pier I, con cargadores con capacidad para $16 \mathrm{mil}$ ton/hora y pudendo recibir buques hasta 400 mil toneladas para porte bruto.

A través del uso de la simulación computacional, software Arena 12.0, se pretende simular la operación del nuevo Pier para cargamento de mineral de hierro y manganeso.

Figura 5 - Modelo del Pier IV

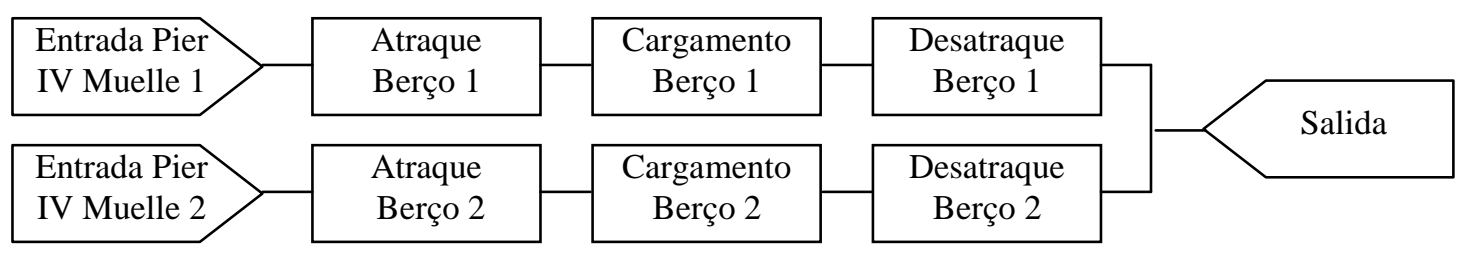

En la aplicación de la Teoría de Colas se utilizó el modelo M/M/1 con atendimiento obedeciendo a la distribución de la Exponencial Negativa. Aunque, muy utilizado en la Teoría de Colas, este modelo no dimensiona colas correctamente, existiendo otros modelos que presentan mejores resultados. El Arena permite el uso de diversos tipos de distribución de frecuencia, tal como mostrado en la Tabla 9.

Tabla 9 - Modelo del Pier IV

\begin{tabular}{|c|c|c|l|}
\hline Distribución & Abrev. & Parámetros & \multicolumn{1}{|c|}{ Mejor aplicación } \\
\hline Poisson & POIS & Media & Llegada \\
Exponencial & EXPO & Media & Llegada \\
Triangular & TRIA & Min/Media/Max & Atendimiento (aproximación inicial) \\
Uniforme & UNIF & Min/Media/Max & Atendimiento (aproximación inicial) \\
Normal & NORM & Media/Desvio & Atendimiento (tiempos de máquina) \\
Johnson & JOHN & G, D, L, X & Atendimiento \\
Log Neperiano & LOGN & Media Logarítmica & Atendimiento \\
Weibull & WEIB & Beta, Alfa & Atendimiento (tiempo de vida de \\
& equipamientos) \\
Discreta & DISC & P1, V1, .. & Llegada/Atendimiento \\
Continua & CONT & P1, V1, .. & Llegada/Atendimiento \\
Erlang & ERLA & Media/K & Atendimiento \\
Gamma & GAMM & Beta, Alfa & Atendimiento (tiempos de reparo) \\
\hline
\end{tabular}

Fuente: Prado (2008, p. 70).

De esta forma, para mejor dimensionar el sistema en análisis se utilizo el modelo $\mathrm{M} / \mathrm{Ek} / 1 / \infty / \infty / \mathrm{FIFO}$, en que: Llegadas siguen Poisson y Atendimientos siguen la Distribución Erlang de grado $\mathrm{k}$.

Para simular la operación del Pier IV se utilizó los datos colectados de atraques y desatraques del Pier I ya que tiene características similares al Pier que será construido. A través de la simulación en el software Arena se obtuvieron los siguientes resultados demostrados en la Tabla 10. 
Tabla 10 - Resultados Obtenidos con la Simulación

\begin{tabular}{|c|c|c|c|c|c|c|c|c|c|}
\hline \multirow{2}{*}{} & \multicolumn{9}{|c|}{ Buques de gran porte } \\
\cline { 2 - 11 } & \multicolumn{2}{|c|}{$100 \%$} & \multicolumn{3}{c|}{$90 \%$} & \multicolumn{2}{c|}{$80 \%$} & \multicolumn{2}{c|}{$75 \%$} \\
\cline { 2 - 10 } & \multicolumn{9}{|c|}{ Buques operados } \\
\hline Modelo & Año & Mes & Año & Mes & Año & Mes & Año & Mes \\
\hline $\mathrm{M} / \mathrm{M} / 1 / \infty / \infty / \mathrm{FIFO}$ & 456 & 38 & 507 & 42 & 577 & 48 & 624 & 52 \\
\hline $\mathrm{M} / \mathrm{E} 2 / 1 / \infty / \infty / \mathrm{FIFO}$ & 461 & 38 & 509 & 42 & 574 & 47 & 637 & 53 \\
\hline $\mathrm{M} / \mathrm{E} 5 / 1 / \infty / \infty / \mathrm{FIFO}$ & 456 & 38 & 497 & 41 & 563 & 46 & 638 & 53 \\
\hline
\end{tabular}

Al analizar los resultados obtenidos con la simulación del Pier IV tiene que esto solo será capaz de operar un total de 53 buques por mes, conforme previsto en su plano de construcción, si hasta $75 \%$ de la su capacidad fuere utilizada para buques de gran porte. Actualmente el Pier I concentra toda la demanda de los grandes buques por ser el único con capacidad de cargar buques con más de 200.000 toneladas de porte bruto. Con la entrada del Pier IV, serán tres muelles que podrán cargar los grandes graneleros. De esta forma, entiende que habrá una distribución de buques entre estos muelles, que no cargarán apenas buques de gran porte, más atendieron también las demandas de buques de pequeño y medio porte. Aconteciendo esta mejor distribución tanto el Pier IV cuanto el Pier I tendrán aumento significativo en el número de buques que pasarán por el sistema.

A través del uso del simulador Arena se puede generar las principales medidas de desempeño del sistema en análisis, tales como las presentadas en la Tabla 11.

Tabla 11 - Informe Arena para el modelo del Pier IV

\begin{tabular}{|c|c|}
\hline \multicolumn{2}{|c|}{ Informe Arena - Pier IV } \\
\hline Tiempo Medio en la Cola (TF) & $\begin{array}{c}3,77 \\
\text { días }\end{array}$ \\
\hline Tiempo en la Fila - Valor Mínimo & $\begin{array}{c}0,00 \\
\text { días }\end{array}$ \\
\hline Tiempo en la Fila - Valor Máximo & $\begin{array}{c}14,80 \\
\text { días }\end{array}$ \\
\hline Tamaño Medio de la Cola (NF) & $\begin{array}{c}4,79 \\
\text { días }\end{array}$ \\
\hline Tamaño da Cola - Mínimo & $\begin{array}{c}0,39 \\
\text { días }\end{array}$ \\
\hline Tamaño da Cola - Máximo & $\begin{array}{c}15,97 \\
\text { días }\end{array}$ \\
\hline $\begin{array}{c}\text { Cuantidad de buques que entraron en } \\
\text { el sistema }\end{array}$ & 648 \\
\hline $\begin{array}{c}\text { Cuantidad de buques que salieron del } \\
\text { sistema }\end{array}$ & 638 \\
\hline
\end{tabular}

\begin{tabular}{|c|c|c|}
\hline \multicolumn{2}{|c|}{ Informe Arena detallado por Entidad (en días) } \\
\hline Variables & $\begin{array}{c}\text { Muelle } \\
\text { I }\end{array}$ & $\begin{array}{c}\text { Muelle } \\
\text { II }\end{array}$ \\
\hline Cuantidad en uso & 0,85 & 0,93 \\
\hline Tasa de Utilización & 0,85 & 0,93 \\
\hline $\begin{array}{c}\text { Número de veces que el recurso } \\
\text { fue utilizado }\end{array}$ & 311,00 & 329,00 \\
\hline Tamaño Medio de la Cola (NF) & 1,87 & 4,79 \\
\hline Tempo Medio en la Cola (TF) & 2,19 & 5,26 \\
\hline
\end{tabular}

\section{Conclusión}

Con los resultados obtenidos, después de la aplicación de la Teoría de Colas, se puede ver la eficiencia de la Terminal Marítima de Ponta da Madeira para embarque de mineral de hierro y manganeso. Los tres muelles destinados al cargamento de buques con estos minerales presentan alta 
tasa de utilización, encima del $80 \%$. De esta forma, para garantir la demanda creciente, principalmente de la Asia y del Oriente Medio, la Vale precisará invertir en la ampliación de la capacidad de la terminal.

De acuerdo con la empresa minera serán invertidos R \$ 2 mil millones en la construcción de un nuevo Pier que elevará la capacidad de la terminal en 100 millones de toneladas al año y tendrá capacidad de cargar un total de 53 buques al mes.

El Pier IV tendrá características operacionales parecidas con el Pier I, con cargadores de 16 mil toneladas/hora, profundidad mínima de 25 metros y capacidad para cargar buques hasta 400 mil toneladas de porte bruto (TPB).

A través de la Teoría de la Simulación y del uso del simulador Arena 12.0 darse cuenta que para cargar 53 buques al mes el nuevo Pier, deberá recibir también las demandas de buques de pequeño y medio porte. Si el nuevo Pier operar sólo buques de gran porte, esto cargará aproximadamente 38 buques al mes. Para cargar 53 buques, como preterido por la mineradora, el Pier IV tendrá que operar un máximo de $75 \%$ dos grandes graneleros.

Esto será posible, pues, con la entrada del nuevo Pier, la demanda por mineral de los grandes buques que antes era concentrada en lo Pier I, por ser el único con capacidad de recibir buques de más de 200.000 TPB, será distribuida para los dos nuevos muelles con capacidad para operar tales buques, en el Pier IV.

De esa forma, el Pier I también se beneficiará con la construcción del Pier IV, teniendo un aumento significativo del número de buques que pasarán por el sistema, una vez que los buques de gran portes serán más bien distribuidos entre los tres muelles.

Con la entrada en operación del Pier IV, la Terminal Marítima de Ponta da Madeira tendrá 5 muelles para cargamento de mineral de hierro y manganeso y 1 muelle (arrendado del Puerto de Itaqui) para cargamento de soya y gusa. Con estas inversiones en ampliación la TMPM será no sólo el mejor más el mayor puerto del Brasil.

\begin{abstract}
Among the available techniques used in key processes of analysis and resolution of outstanding problems of queuing theory and simulation theory: that an analytical method to approach the matter, this, a modeling technique that seeks to better represent the system study. Using these modeling techniques for complex problem solving and dynamic, has grown significantly in recent years, though, there is a perceptible gap in the literature of applications in real situations. In contribution, this work explores the use of queuing theory and simulation applied to the shipment of iron ore and manganese in the maritime terminal of Ponta da Madeira - TMPM, owned by Vale. Queuing Theory With one tries to analyze characteristics of services rendered to vessels berthing at Pier Pier I and III for shipment of iron ore and manganese, such as average number of vessels in the queue and the system, average time for which the vessel remains in the queue and the system etc. And with the technique of simulation is to simulate the operation of the Pier IV, which consume R \$ 2 billion investment, will increase the terminal capacity by 100 million tons per year and will be able to load 53 ships per month.
\end{abstract}

Key-words: queuing theory; simulation; TMPM. 


\section{Referencias}

ABAD, R. C. Introducción a la simulación y a la teoría de colas. London: Netbiblo, 2002.

ANDRADE, E. L. Introdução à pesquisa operacional: métodos e modelos para análise de decisões. 3. ed. Rio de Janeiro: LTC, 2004. 192 p.

BANKS, J.; CARSON, J. S.; NELSON, B L.. Discrete-event system simulation. 2. ed. New Jersey: Prentice Hall, 1999.

BROWN, L. et al. Statistical analysis of a telephone call center: a queueing-science perspective. Wharton Financial Institutions Center, Working Paper, 03 - 12, Nov 9, 2002.

BRUIN, A. M.; KOOLE, G. M.; VISSER, M. C.. Bottleneck analysis of emergency cardiac in-patient flow in a university setting: an application of queueing theory. Clinical and Investigative Medicine, v. 28, p. 316-317, 01 jul. 2005.

COSTA, L. C. Teoria das Filas. Disponible en: <http://www.deinf.ufma.br/ mario/grad/filas/TeoriaFilas_Cajado.pdf> . Acceso en 20 out. 2009.

FARRERO, J. M. C.; TARRÉS, L. G. La empresa de servicios y la teoría de colas. New York: Uoc/digitalia, 2009.

GREEN, L. V.. Using queueing theory to increase the effectiveness of emergency department provider staffing. Academic Emergency Medicine, v. 13, p. 61-68, 01 jan. 2006.

GROSS, D.; HARRIS, C. M.. Fundamentals of queueing theory. 2. ed. New York: John Wiley \& Sons, 1985.

JOSHI, P. L. et al. Application of queueing theory in hospital management. Indian journal of community medicine. Allahabad, v. 17, p. 102-108, 01 jul. 1992.

MIRSHAWKA, V. Pesquisa operacional. 3. ed. São Paulo: Nobel, 1979.

MOORE, B. J.. Use of queueing theory for problem solution in Dallas, Tex., Bureau of Vital Statistics. Public health reports, v. 92, p. 171-175, 01 jan. 1977.

REDONDO, R. P. D.; ARIAS, J. J. P.; GONZALEZ, A. S. Teoría de colas y simulación de eventos discretos. London: Pearson, 2004.

MIRANDA, M. Teoría de colas. Buenos Aires: Educa, 2005.

O CUSTO da ineficiência. PORTAL EXAME. en: <http://portalexame.abril.com.br/revista/exame/edicoes/Infra2008/especiais/custo-ineficiencia-410882.html>. Acceso en: 30 set. 2009.

O RISCO do apagão portuário. PORTAL EXAME. en: <http://portalexame.abril.com.br/static/aberto/infraestrutura/edicoes_2007/m0143169.html>. Acceso en: 01 out. 2009.

PORTUGAL, L. da S. Simulação de tráfego: conceitos e técnicas de modelagem. Rio de Janeiro: Interciência, 2005.

PRADO, D. S. do. Teoria das filas e da simulação. Nova Lima (MG): INDG, 2006.

Usando o arena em simulação. Belo Horizonte: INDG, 2008.

RANKING expõe deficiências e deixa Porto de Santos entre piores do País. ABRETI. Disponível em: <http://abreti.org.br/news/30_07_07.htm>. Acceso en: 4 nov. 2009.

TERMINAL marítimo de Ponta da Madeira é o melhor do Brasil. VALE. Disponible en: <http://www.vale.com/saladeimprensa/pt/releases/release.asp?id=17661>. Acceso en: 4 nov. 2009.

XIAO, H.; ZHANG, G. The queuing theory application in bank service optimization. In. INTERNATIONAL CONFERENCE ON LOGISTICS SYSTEMS AND INTELLIGENT MANAGEMENT, 24. 2010, Harbin. Anais... Harbin: ICLSIM, 2010. 


\section{Dados dos autores:}

Nome completo: Gustavo Rossa Camelo

Filiação Institucional: Universidade Federal de Santa Catarina

Departamento: Engenharia de Produção - DEPS/UFSC

Função ou cargo ocupado: Mestrando em Engenharia de Produção (Bolsista CNPq)

Endereço para correspondência (bairro, cidade, estado, país e CEP): Marcus Aurélio Homem, 250, Apto. 7 - Trindade, CEP: 88040440

Telefones para contato: (48) 3721-7313 / 96294689

e-mail: gustavorcamelo@hotmail.com

Nome completo: Renata Massoli Borges

Filiação Institucional: Universidade do Sul de Santa Catarina

Departamento: Administração

Função ou cargo ocupado: Mestranda em Administração

Endereço para correspondência (bairro, cidade, estado, país e CEP): Servidão Dionizio Raphael Ignácio, 99, Apto 302 - Campeche, CEP: 88063315

Telefones para contato: (48) 99051431

e-mail: renata@fama.br

Nome completo: Antônio Sérgio Coelho

Universidade Federal de Santa Catarina

Departamento de Engenharia de Produção - DEPS/UFSC

Professor Adjunto do Programa de Pós-Graduação em Engenharia de Produção

Endereço para correspondência (bairro, cidade, estado, país e CEP): Deputado Antônio Edu Vieira, 434, Parco Reale, Bloco B, Apto. 102 - Pantanal, CEP: 88040-000

Telefones para contato: (48) 3721-7058

e-mail: coelho@deps.ufsc.br

Recebido para publicação em: 27/08/2010

Aceito para publicação em: 05/10/2010 